\title{
Multi-Constraints based RPL objective function with adaptive stability for high traffic IoT applications
}

\author{
Abdelhadi Eloudrhiri Hassani, Aicha Sahel, Abdelmajid Badri, El Mourabit Ilham \\ EEA\&TI laboratory, Faculty of Sciences and Techniques, Hassan II University, Casablanca, Morocco
}

Article Info

Article history:

Received Oct 9, 2020

Revised Jan 23, 2021

Accepted Mar 3, 2021

Keywords:

Combined metrics

Contiki OS

RPL

Workload balancing

WSN

\begin{abstract}
The internet of things technology is classified as a Low power and lossy network. These kinds of networks require a trustworthy routing protocol considered as the backbone for management and high quality of service achievements. IPv6 Routing Protocol for Low power and lossy network (RPL) was able to gain popularity compared to other routing protocols dedicated to IoT for its great flexibility through the objective function. Default objective functions implemented in the RPL core are based on a single metric. Consequently, the routing protocol can't cope with different constraints and show congestion issues in high traffics. For that, we proposed in our paper Multi-Constraints-based Objective Function with Adaptive Stability (MCAS-OF), which uses novel strategies for Radio strength indicator, node energy consumption, hop count and a designed work-metric combination, new rank processing, and parent selection procedure. The network stability was also taken into account, since the multi constraints can lead to frequent parent changes, using an adaptive threshold. The proposal, evaluated under the COOJA emulator against Standard-RPL and EC-OF, showed a packet delivery ratio improvement by $24 \%$ in high traffics, a decrease in the power consumption close to $44 \%$, achieved less latency and DIO control messages, it also gives a good workload balancing by reducing the standard deviation of node's power consumption.
\end{abstract}

This is an open access article under the $\underline{C C B Y-S A}$ license.

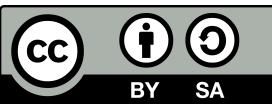

\section{Corresponding Author:}

Abdelhadi Eloudrhiri Hassani,

EEA\&TI laboratory,

Faculty of Sciences and Techniques, Hassan II University, Morocco,

Email: eloudrhiri.abdelhadi@gmail.com

\section{INTRODUCTION}

IoT, considered as a great connectivity potentiel for humanity, are tiny sensor devices [1, 2] based networks with constraint and limited resources [3]. Consequently, there is a need for appropriate resources management and utilization to cope with different IoT applications and fields as smart grids [4, 5], smart cities [6], industries [7] and healthcare [8]. Thereby, in order to ensure perfomances efficiency of those networks, the researchers have been interested mostly in routing protocols dedicated to IoT applications. In this context, the most commonly is RPL designed by Internet Engineering Task Force (IETF) [9].

RPL is based on IPv6 and uses the IEEE802.15.4 at the Physical (PHY) and Medium access control (MAC) layers [10]. Packets based on IPv6 cannot fit in the IEEE 802.15.4 protocol. For this reason, 6LowPAN, IPv6 Low Power Personal Area Network layer [11], acts as an adaptation between MAC and network layers. With RPL, the paths are constructed once the network is initialized. The nodes aims to set up destinationoriented directed acyclic graph (DODAG), a tree routing topology, using four principal ICMPv6 messages : 
DODAG Information Object (DIO) holds informations that enable nodes to know the instance, configuration and preferred parent, it managed by the trickle algorithm and necessary for Multiple Points to Point (MP2P) and Point-to-Point (P2P) communications [12]. DODAG Information Solicitation (DIS) message as a DIO request from neighbours. DODAG Advertisement Object packets (DAO) used to collect downward topology informations and DAO-ACK as a response to a DAO message. The aim of DODAG topology is to steer data packets to one or multiple sink. Routing paths are created using an objective function (OF). In the core of RPL, two OF's are proposed namely the minimum rank with hysteresis objective function (MRHOF) [13] based on expected transmission counts (ETX) as a routing metric and OF0 [14], which is based on hop count. The paths in MRHOF are based on the link quality metric calculated by broadcasting probe packets between the sender and receiver nodes at time intervals. On the other hand, the routing paths in OF0 are based on node metric that aims to each node in the network to know its position related to sink. However, the two OF tend to minimize the cost of their metrics which causes non-optimized routes due to taking into account a single constraint. Furthermore, they are not effective for high traffic applications and load balancing between network nodes. Considering these drawbacks, we have proposed Multi-Constraints based objective function with Adaptive Stability (MCAS-OF), which uses novel strategies for link and node metrics combination, new rank processing and parent selection procedure. The network stability issue is also taken into account since the multi constraints can lead to frequent parent changes. Most of the researchers induce a constant threshold, consequently, it decreases the chances for nearest nodes from sink to change their parents even if they are congested or have a lot of workload. For that purpose, our objective function uses an adaptive threshold based on ranks. The main contributions of this paper are summarized as follows:

a. Improve the RPL QoS by a newly designed objective function MCAS. Our proposal combines multimetrics chosen to cope with different constraints while respecting the objective function convergence.

b. Consider the network stability in routing by a proposed adaptive threshold based on rank's node.

c. A simulation under COOJA of MCAS-OF compared to Standard-RPL and EC-OF in term of PDR, Energy consumption, Latency, DIO control messages and standard deviation.

The rest of this paper is organized as follow. In Section 2. we present the related works with RPL routing protocol. In Section 3. we present the proposed objective function MCAS. In Section 4. we report the performance evaluations results and discussion, finally a conclusion is given in Section 5 .

\section{RELATED WORKS}

In this section, we present some related researches to our work that improve the objective functions performances for RPL protocol in different IoT applications. In [15], authors proposed an extented metric based on the expected transmission count called Sigma-ETX which is the standard deviation value of ETX by number of hops to sink. This technique avoid the long hops issue that cause bottlenecks in high network densities. Also ELT metric, i.e. Expected Lifetime, was designed by authors in [16] that consist of the node remaining lifetime before to be out of service. It is injected in RPL based on multipath, which leads to enhance the network reliability despite an additional delay induced. Node's remaining energy metric was designed by authors in [17] on which the objective function is based to select preferred parents. This proposal has proved its ability to improve the overall network lifetime but doesn't cope with the reliability since it can choose lossy links. Minimizing the delay of receiving packets by the sink was considered by authors in [18] using the AVGDELAY as a routing metric. This technique, ran with a low duty MAC cycle, showed a decrease in terms of end-to-end delay but don't address the network reliability at all. The fuzzy logic approach was considered by authors in [19]. Indeed, they proposed COOF, i.e. a novel objective function context-oriented objective function, that considers two designed metrics respectively remaining energy (RE) and queue fluctuation index (QFI) for smart cities application based IoT requirements. Using the same approach in [20], the authors proposed fuzzy objective function called EC-OF. It combines ETX and node energy consumption while hop count as node redirection metric. Results showed that the proposal improves the RPL performances in terms of packet delivery ratio, latency, convergence, power consumption including the network lifetime against MRHOF. Similarly, the authors in [21] proposed DQCA-OF that combines the same three metrics considering different performances. The method showed a significant decrease in delay and a high PDR in low density. The authors in [22] proposed Fuzzy Logic Based Energy Aware (FLEA) for RPL objective function based on residual node energy, expected 
transmission count ETX and traffic load. it is used for calculating the rank increase parameter for node's rank. The proposal shows a increase of PDR around 2\% to 5\% and lifetime around 10\%. The context-awareness is also considered during novel objective functions proposal. In [23], authors designed Scalable Context-Aware for Agricultural environmental monitoring. SCAOF is based on energy, reliability, resource, and robustness routing metrics. The proposal Performances, verified using real and simulation tests, can provide the desired advantages in terms of reliability, high efficiency, and network lifetime extension. Congestion problem due to the high number of forwarded packets was addressed in [24] by proposing Forwarding Traffic Consciousness objective function, which combines hop count, rssi and a newly designed FTM metric. The proposed method showed a packet delivery ratio increase respectively with $2 \%$ and $11 \%$ in low and high traffics, considerably reduces the power consumption with approximately $47 \%$ as well as it achieves a good balance of traffic managed by the relay nodes. The additive and lexical approachs were also the base of several objective function optimizations. Indeed, authors in [25] proposed a new objective function based on additive combination of node and link metrics respectively ETX and Energy consumption along the routing paths. They proposed two way of combination, i.e. with weights and non weighted methods. Results show that the WCMOF and NWCMOF can increase the reliability, maximize the network life time and reduce the parent changes. Smart grid application requirements were considered by authors in [26]. The authors designed OFQS objective function based on power state metric, expected transmission count and delay. The stability was considered by the hysteresis concept as in MRHOF. Results showed improvement in terms of end-to-end delay, network lifetime and packet delivery ratio. The authors proposed lexicographic and additive approaches in [27] to combine expected transmission count, hop count and available energy metrics in EHAOF. The results showed better performance in terms of energy consumption, network latency and packet delivery ratio against MRHOF-ETX and OF0. In [28], authors have been interested in the congestion of the path caused by the buffer nodes occupancy. For that purpose, Congestion-Aware Objective Function CA-OF were be proposed which consider ETX metric at a low data rate while the buffer occupancy is considered at a high data rate. Figure 1 [29] summarizes the structure of different researches related to RPL protocol.

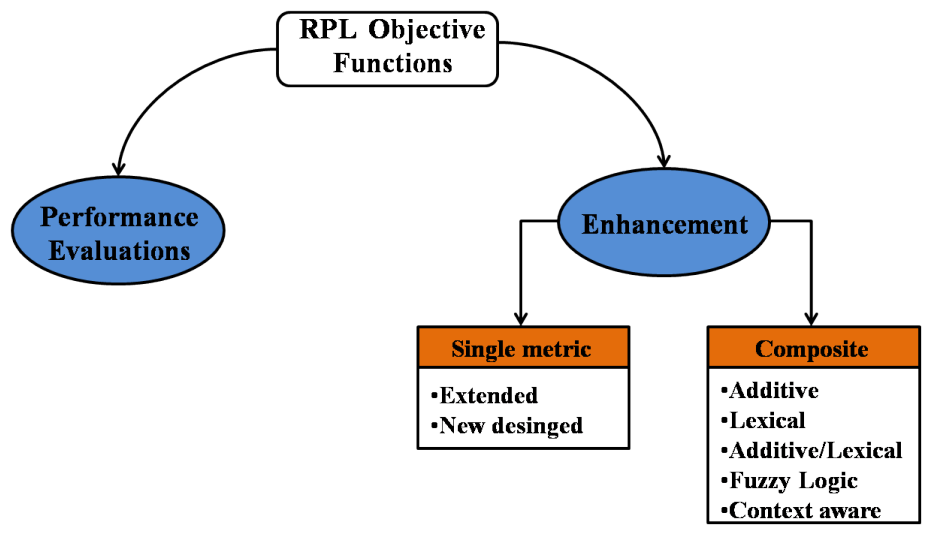

Figure 1. Different researches related to RPL protocol

\section{RESEARCH METHOD}

The QoS offered by a routing protocol for IoT based on wireless sensor networks is one of the substantial design concerns. Besides, its multiple requirements meeting allows it to be widely used for different applications. As mentioned before, the RPL objective function is mainly responsible for satisfying the application prerequisites according to the constraint metrics used in the preferred parents selection. These metrics are commonly classified into two categories. The first category considers the quality of links between nodes as rssi, delay and expected transmission count ETX, while the second category regroups node parameters such as power consumption, residual energy, number of hops, queue utilization. In a standard manner, RPL employs an objective function with one metric to minimize. This lead to cope with limited constraints, which certainly inflict poor performances in term of various requirements. For instance, the objective function based on expected transmission count ETX can show low packet losses, but ignores the node's energy consumption management. Also, based on the hop count metric, the RPL routing protocol can minimize the hops to the destination then 
achieves a high level of energy preservation but can't cope with the network reliability. Furthermore, the objective functions defined in the RPL core are not optimized for high traffic applications and considering load balancing between network nodes. To overcome these challenges, we have proposed Multi-Constraints based objective function with Adaptive Stability (MCAS-OF), which uses novel strategies for link and node metrics combination, new rank processing and parent selection procedure. The network stability issue is also taken into account since the multi constraints can lead to frequent parent changes. Most of the researchers induce a constant threshold, consequently, it decreases the chances for nearest nodes from sink to change their parents even if they are congested or have a lot of workload. For that purpose, our objective function uses an adaptive threshold based on ranks.

\subsection{Multi-Constraint Metrics with Workload Consideration}

Reliability, energy consumption, workload management, shortest path are the most influential parameters on the QoS of IoT applications. Accordingly, it is important to determine how these four parameters should be combined to make the best routing decisions. In this context, we have proposed in our paper an additive combination of four metrics namely rssi, node energy consumption, hop count and a designed work-metric. However, these metrics should be chosen in such a way that all must be minimized to avoid loops in routing and respect the objective function convergence.

Number of hops is the only one among the four metric which is announced by neighboring nodes. For that purpose, we have modified the structure of the DIO control message precisely in the metric container part. Indeed, at reception of DIO message by a node i, it process the hop count metric following Equation 1.

$$
H C(i)= \begin{cases}\text { Minhc_Increment } & \text { if } n=\operatorname{sink} \\ h c(n)+\text { Minhc_Increment } & \text { if } n \neq \operatorname{sink}\end{cases}
$$

Where $\mathrm{n}$ is the neighbor susceptible to be the preferred parent of node $\mathrm{i}, \mathrm{HC}(\mathrm{n})$ reprensent the number of hops to the sink advertised in the neighbor DIO whereas MinHC_Increment is a scalar value equal to 256.

Energy consumption is calculated by a node i using the power trace tool following Equation 2, it is the summation of transmission, reception, $\mathrm{cpu}$, and $\mathrm{lpm}$ power consumptions in each state.

$$
E C(i)=\frac{T \_c p u * 5.4+T_{\_} t x * 58.5+T_{\_} r x * 64.5+T_{-} L P M * 0.1635}{R T I M E R \_A R C H \_S E C O N D} * \text { Voltage }
$$

Where T_cpu, T_tx, T_rx, T_LPM are respectively the ticks numbers when the node is processing at the cpu level, transmitting, listening or going to low power mode followig the MAC protocol, the numerical parameters are nominal values provided in the Skymote datasheet, RTIMER_ARCH_SECOND represents the number of ticks per second equal to 32768 and Voltage is the initial battery value equal to $3 \mathrm{~V}$.

Work-metric is processed following Equation 3, such as it the summation of the number of sent data packets and the number of DAO control messages. Indeed, the DAO is unicast from children to the parent node. For that, a node receiving more DAO implies that it manage more nodes. Then, the work-metric gives an idea of the workload of each node, and should be minimizable.

$$
\text { work_metric }(i)=D A T A \_s e n t \_p a c k e t s+D A O \_r e c e i v e d
$$

Counts the number of sent packets at the IP layer. It includes the number of packets containing the data sent by the node $\mathrm{i}$ and the DAO control messages. The sent data are equal between all nodes but the DAO are not. Indeed, when a node has a lot of childrens, it receives more DAO control messages which gives an idea of the number of childrens then the workload imposed to each node. For this reason, we designed this metric that aims to balance the load between all the nodes.

Radio signal strength indicator(rssi) represent a link metric measured by a node $\mathrm{i}$ through the CC2420 radio following Equation 4.

$$
\text { rssi }(i)=\text { rssi_reg }(i)-\text { empirical_adjustment }
$$

Where rssi_reg(i) is a 8 bits register that gives the signal strength between node $\mathrm{i}$ and its neighbor. The antenna variation offset is represented with empirical_adjustment equal to 45 provided from the CC2420 radio datasheet. Since the rssi metric gives values between 0 and $-110 \mathrm{dBm}$ respectively for good and bad signal strength, this metric must be maximized. To keep the objective function convergence, we worked with its absolute value to follow the minimizing rule as other combined metrics. 


\subsection{Designed MCAS Objective Function}

During the process of parent selection, nodes are based on ranks calculated by the objective function. Neighbors with lower rank are elected by the concerned node to be its preferred parent. Unlike standard objective functions based on a single metric, MCAS-OF uses a set of metrics combined for rank processing. Indeed, at a DIO reception from a neighbor, the node i calculates the number of hops, work-metric, measure its energy consumption and the RSSI at the MAC layer. The combination of these metrics is done linearly with different weights following Equation 5.

$$
\text { Combined }(r s s i, \text { energy,work, } h c)=\alpha * \operatorname{rssi}(i)+\beta * \text { energy }(i)+\gamma * \text { work_metric }(i)+h c(i)
$$

The weights are distinguished between constant, complementary, and influencing. In this paper, the hop count weight is related to a unit constant value, rssi, and energy consumption have a complementary weights equal to $1(\alpha, \beta)$ while the work metric have an influencing weight empirically defined $(\gamma)$. Subsequently, the node i extract the rank of neighbor advertised in the DIO message and process its rank according to Equation 6.

$$
\operatorname{Rank}(i, n)=\operatorname{Rank}(n)+\text { Combined }(\text { rssi, energy, work }, h c)
$$

Where Rank(n) is the rank of the neighbor candidate to be the preferred parent through node $\mathrm{i}$ could forward packets to sink. At this stage, once the node has calculated its rank based on the DIO sender, if it is the first round for network establishment, the node chooses it as the preferred parent. Otherwise, a rank comparison between old and candidate parent is done, if it is higher, the candidate parent is discarded. Else, considering the adaptive stability, if it is lower than the preferred parent rank with the adaptive threshold value, then the candidate parent is retained. The threshold is a mandatory parameter for reducing the preferred parent changes. For that, the adaptive threshold defined in Equation 7 is used for MCAS objective function in order to give routing path stability on one hand, and on the other hand, increase the chances for nodes near to sink to change their preferred parent.

$$
\text { Adaptive_Threshold }(i, n)=\frac{\operatorname{rank}(i)+\operatorname{rank}(n)}{2}+\text { Minh__Increment }
$$

Finally, if the condition for the parent change is fulfilled, the node updates its rank and the hop count metric in the DIO message, then broadcast a new one to advertise neighbors that there is a change in the routing path. Algorithm 1 shows the proposed MCAS objective function algorithm implemented in the RPL core, while Figure 2 summarizes the new process of routing decision.

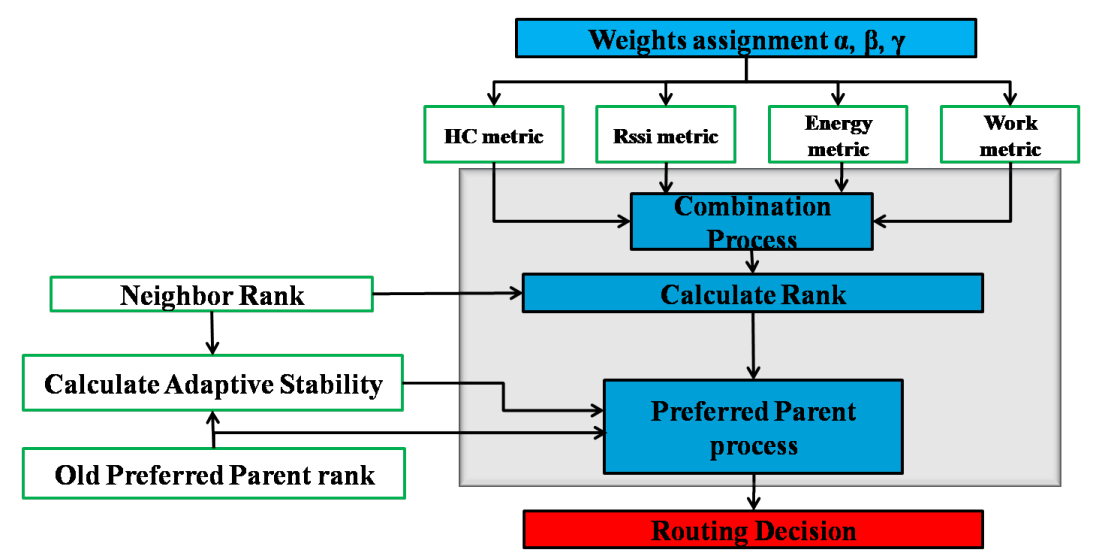

Figure 2. Process of preferred parent selection with MCAS-OF 


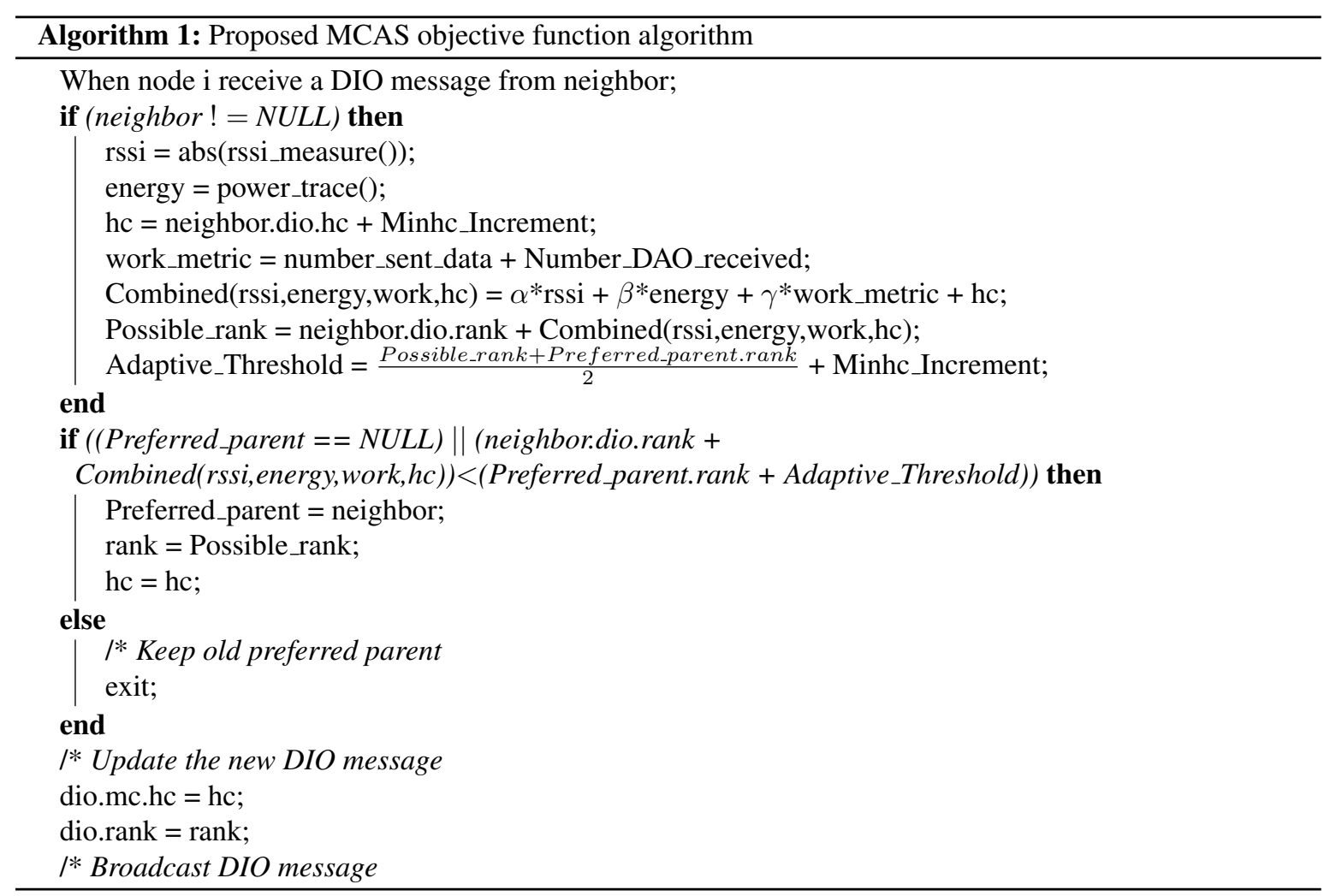

\section{RESULT AND DISCUSSION}

\subsection{Simulation Settings}

The performance evaluation of the proposed objective function is based on simulation carried on the Cooja simulator, using embedded platforms with operating system Contiki 2.7 [30]. Cooja is a simulator/emulator widely used by the IoT researchers to test their proposed works. The network consists of 25 or 50 client nodes with a unique sink. Each node generates a 1 to 20 packets per minute to test our work in multiple scenarios especially in high traffics. Cooja provides as one of the radio models, the UDGM i.e. unit disk graph medium (UDGM), that adds wireless medium losses, which has been used to cope with a realistic simulation environment. The looseness in the medium is relative to the distance between devices in the network. In our scenario, we set 70 meters as the transmission range with 100 meters for the interference range. The nodes are randomly distributed in an area of $200 \times 200 \mathrm{~m}^{2}$, while the sink is placed away from the monitored network. The platforms that are used in the simulation are the Sky mote type, which has MSP430 as a microcontroller with $2.4 \mathrm{GHz}$ wireless transceiver Chipcon CC2420. The motes run Contiki $2.7 \mathrm{OS}$ and conform with communication protocol IEEE 802.15.4. The simulations are performed over 600s for every environmental setup. Simulation settings are summarized in Table 1.

Table 1. Simulation Settings

\begin{tabular}{lc}
\hline Network simulator & Cooja \\
Embedded operating system & Contiki 2.7 \\
Radio environment & Unit disk graph medium - DL \\
Emulated nodes & Sky motes \\
Network area & $200 \times 200 \mathrm{~m}^{2}$ \\
Deployment of nodes & Random \\
Number of sinks & 1 \\
Number of senders & 25,50 \\
Generated packets & $1,5,15,20 \mathrm{ppm}$ \\
Transmission / interference ranges & $70 / 100 \mathrm{~m}$ \\
Simulation time & $600 \mathrm{~s}$ \\
\hline
\end{tabular}

Indonesian J Elec Eng \& Comp Sci, Vol. 22, No. 1, April 2021 : 407 - 418 


\subsection{Performances Evaluation}

\subsubsection{Packet Delivery Ratio}

Figure $3 \mathrm{a}$ and $3 \mathrm{~b}$ show that in low traffic, whatever the density, the three objective functions have almost identical result or a slight difference. But when the traffic increase, MCAS-OF provides an average PDR increase with $5.5 \%$ and $16 \%$ in a density of 25 nodes, $14 \%$ and $24 \%$ in a density of 50 nodes, against Standard-RPL and EC-OF respectively. These results can be explained by the fact that, in high traffic, the congestion in paths frequently occurs with standard objective functions that minimize a single metric.

While our proposal, in addition to the four metrics combination, the work-metric can influence routing path selection by the dispersion of node's workload then decrease the probability of congestion. The other reason is the overall network stability provided by the adaptive stability threshold which limits the frequent parent changes then unnecessary packets loss.

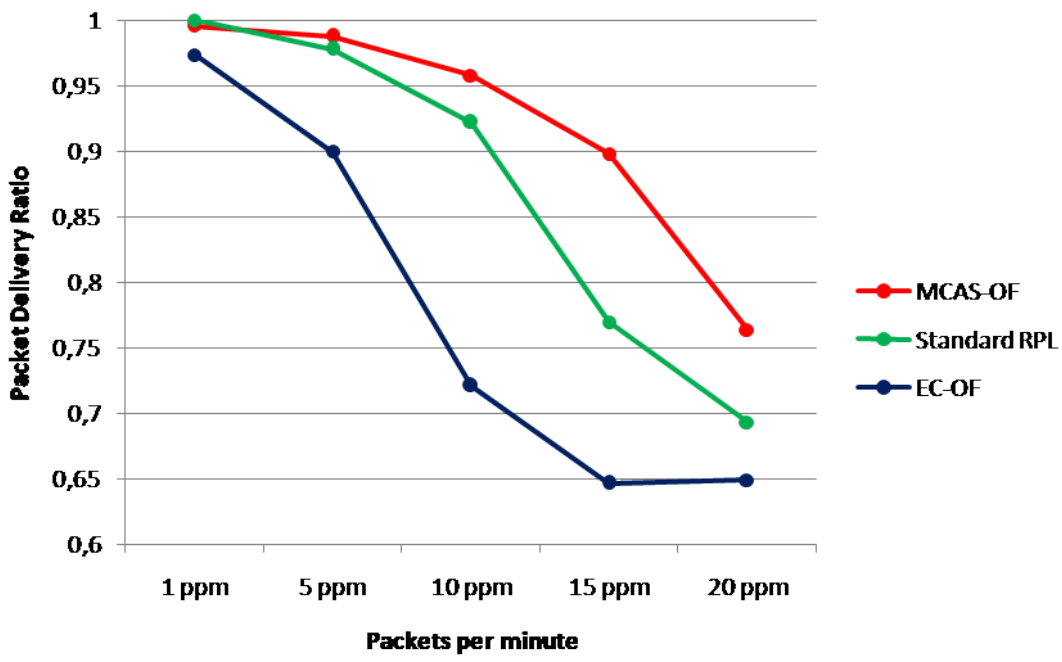

(a)

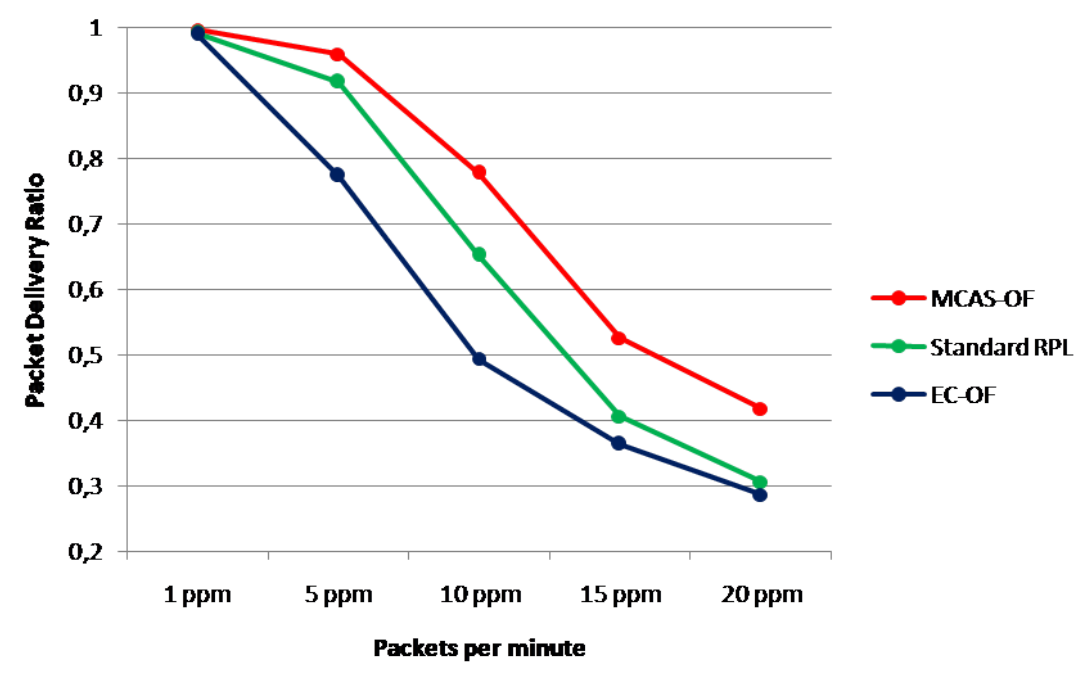

(b)

Figure 3. Packet Delivery ratio vs number of packets per minute, (a) Density of 25 nodes, (b) Density of 50 nodes 


\subsubsection{Average Power Consumption}

In term of node's average power consumption, Figure $4 \mathrm{a}$ and $4 \mathrm{~b}$ show that our proposal consumes less than the other objective functions. Indeed, MCAS-OF decreases the power consumption with $21 \%$ and $32 \%$ in a density of 25 nodes, $40 \%$ and $44 \%$ in a density of 50 nodes, compared to Standard-RPL and EC-OF respectively. The reason for less consumption is the hop count and energy metrics introduced in rank processing, so nodes can avoid unnecessary retransmissions of packets due to long hops while considering also the energy consumption. The other reason is the rssi metric which allows the parent selection where the signal strength between them is good, also it limits energy losses in the packet transmission over poor link quality.

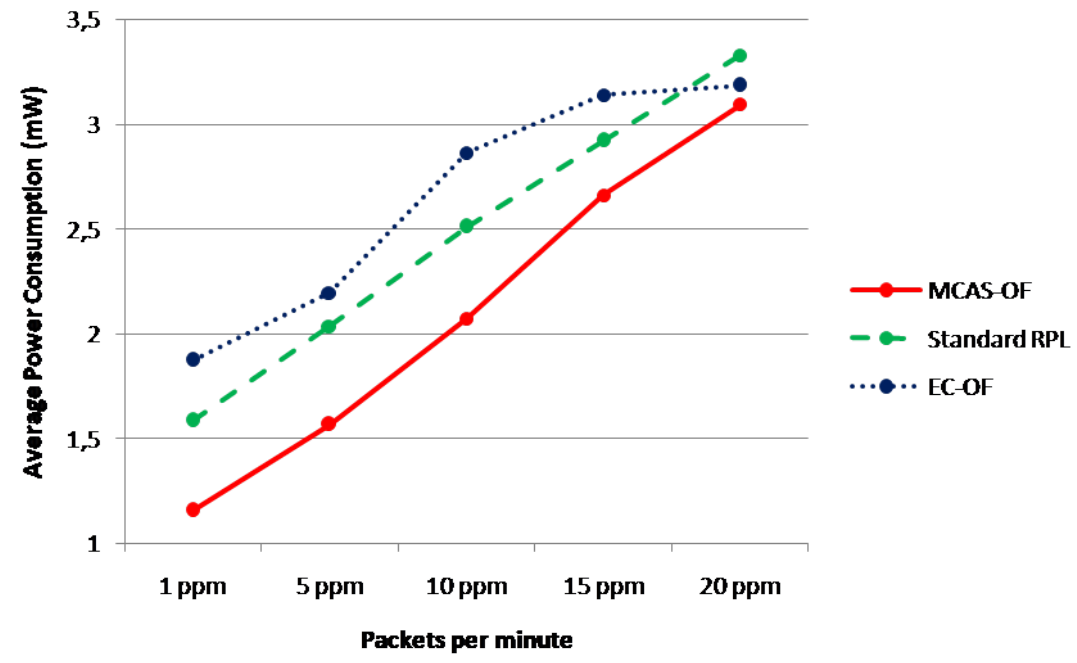

(a)

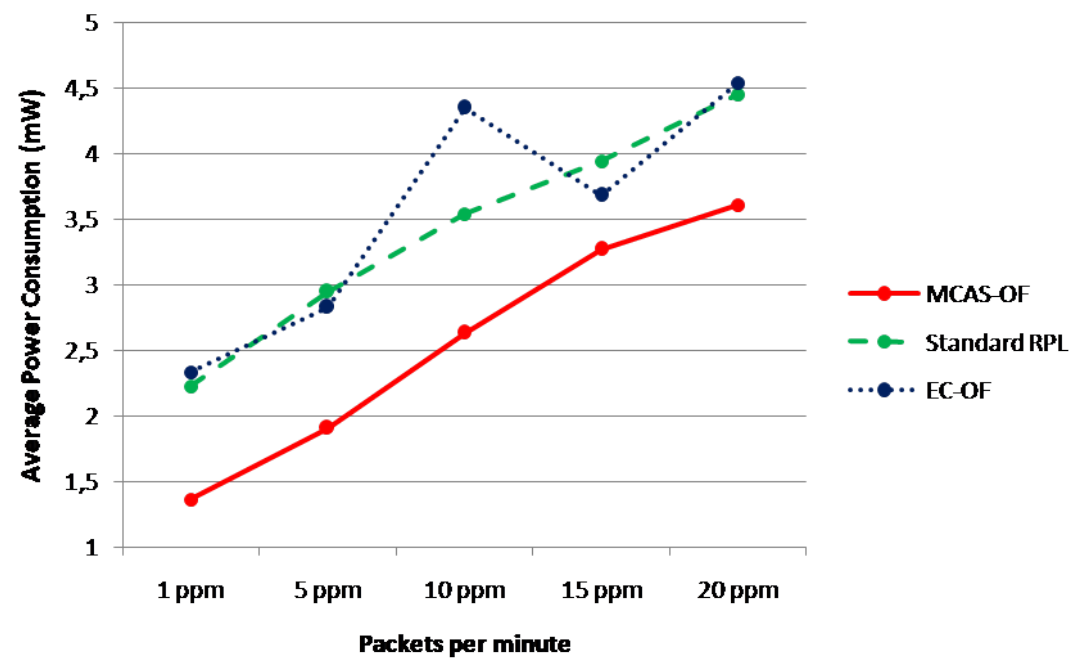

(b)

Figure 4. Average power consumption vs number of packets per minute,

(a) Density of 25 nodes, (b) Density of 50 nodes

\subsubsection{Standard Deviation of Nodes Power Consumption}

The Standard deviation, as a statistical parameter, is included in the performance evaluation to investigate on the power consumption distribution between nodes. It is defined as the deviation of the energy consumption of each in the network related to their average. A glance on Figure 5a and 5b shows that our proposal significantly decreases the difference between the power consumption of nodes and their average.

Indonesian J Elec Eng \& Comp Sci, Vol. 22, No. 1, April 2021 : 407 - 418 
These results can be explained thanks to the work metric introduced in the combined metrics, which fulfills its load-balancing role between all the nodes, even for those close to the sink.

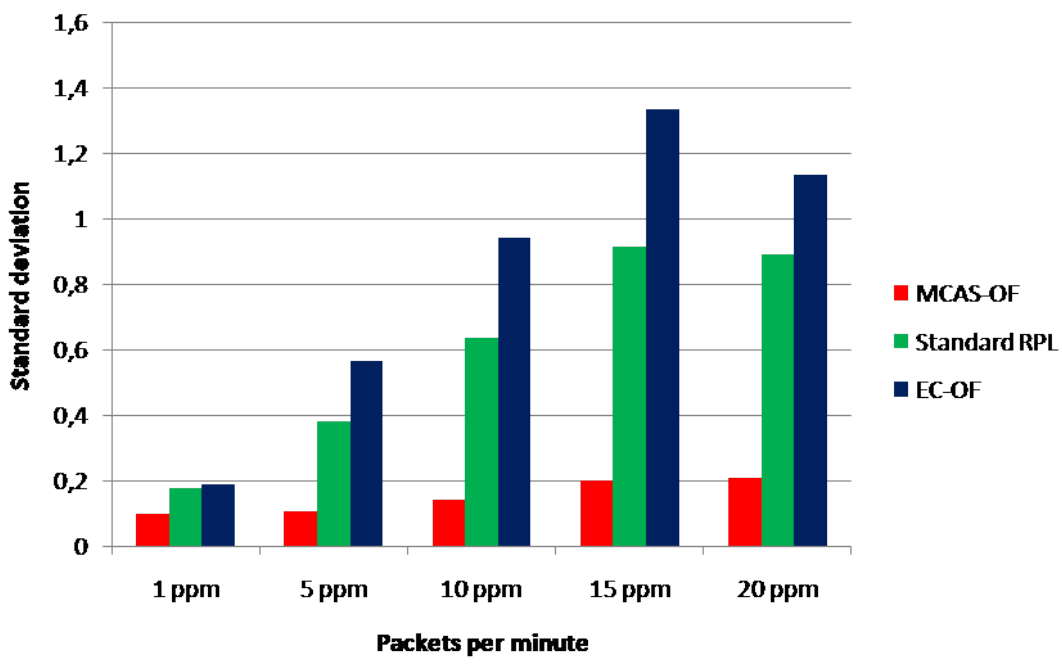

(a)

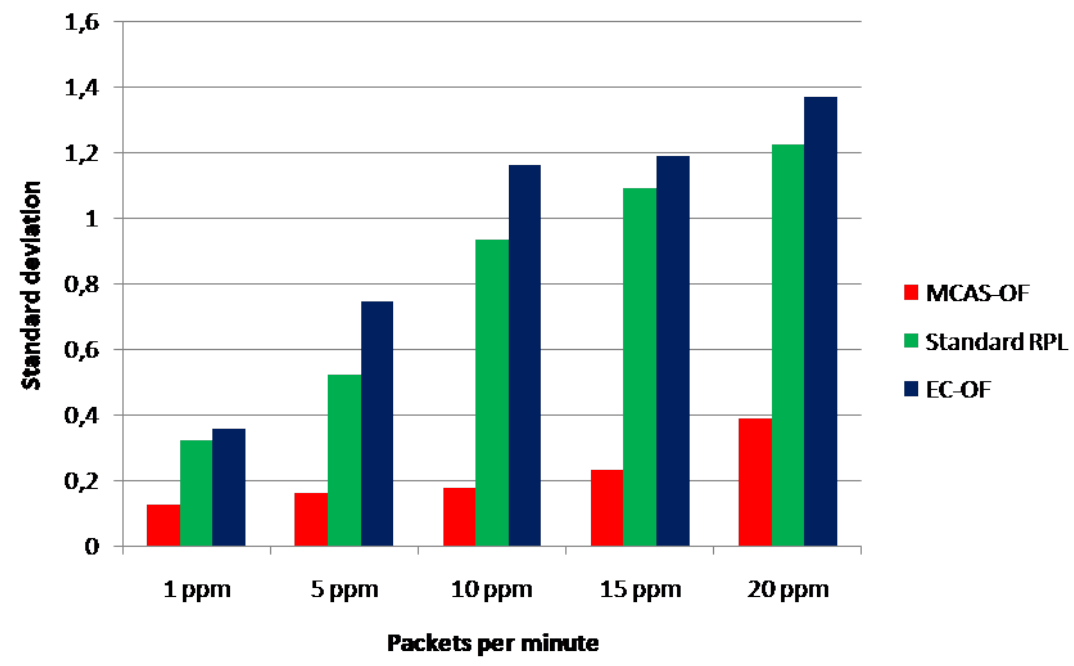

(b)

Figure 5. Standard deviation of nodes power consumption vs number of packets per minute,

(a) Density of 25 nodes, (b) Density of 50 nodes

\subsubsection{DIO Control Packets}

DIO control packets are used by RPL protocol to create and maintains the network topology. As mentionned before, every node broadcasts DIO periodically using the trickle algorithm to update nodes status. More the network is stable, the DIO sent frequency will be reduced. The results in Figure 6a and 6b show that proposed MCAS-OF generates less DIO compared to the other objective functions. This is because the proposal uses the adaptive stability threshold that give more stability in routing paths. 


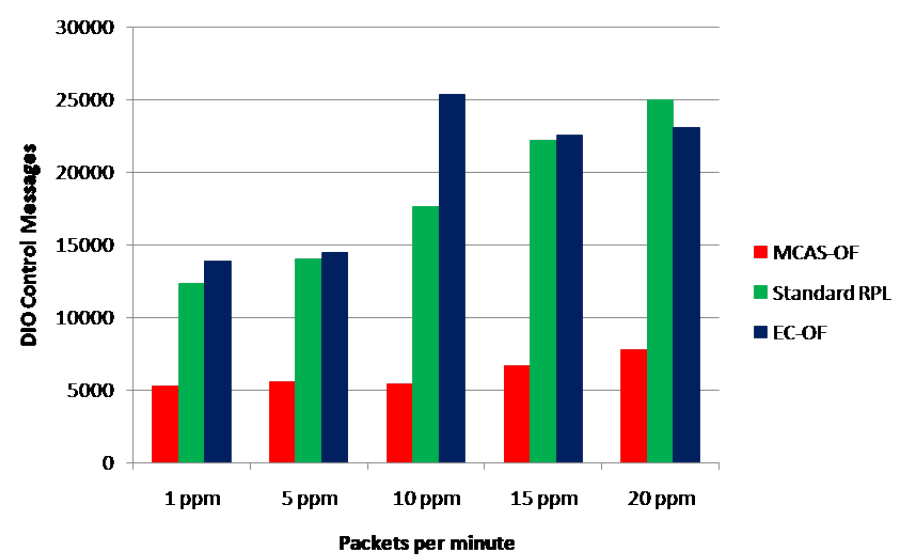

(a)

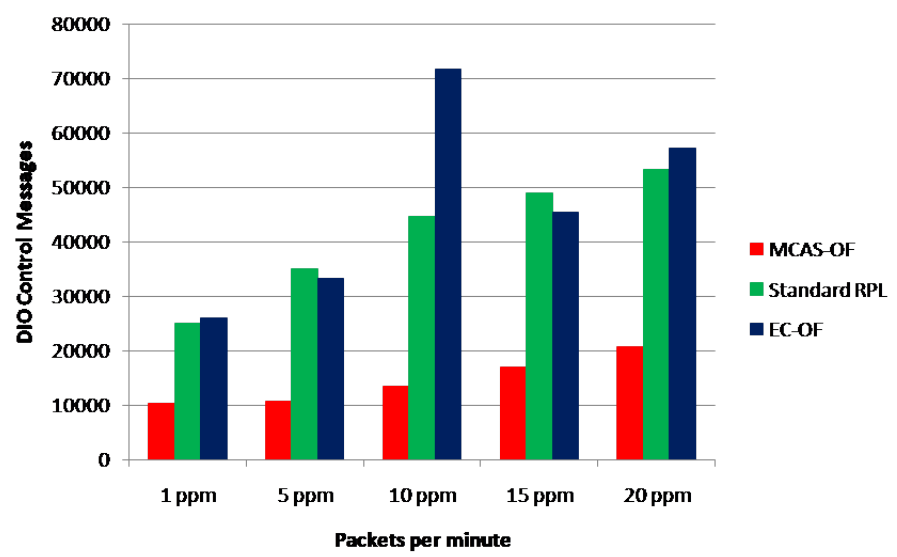

(b)

Figure 6. DIO control packets vs number of packets per minute,

(a) Density of 25 nodes, (b) Density of 50 nodes

\subsubsection{Latency}

Latency is defined as the total delay that make sent packets by a node in the network to be succesfully received by the sink. Compared to other objective functions taken in our performances evaluation, MCAS-OF gives less delay duration as shown in Figure $7 \mathrm{a}$ and $7 \mathrm{~b}$ especially when the number of packets sent per minute increase. The results can be explained by that our method can relieve congestion that may occur in high traffics, allowing for faster package delivery.

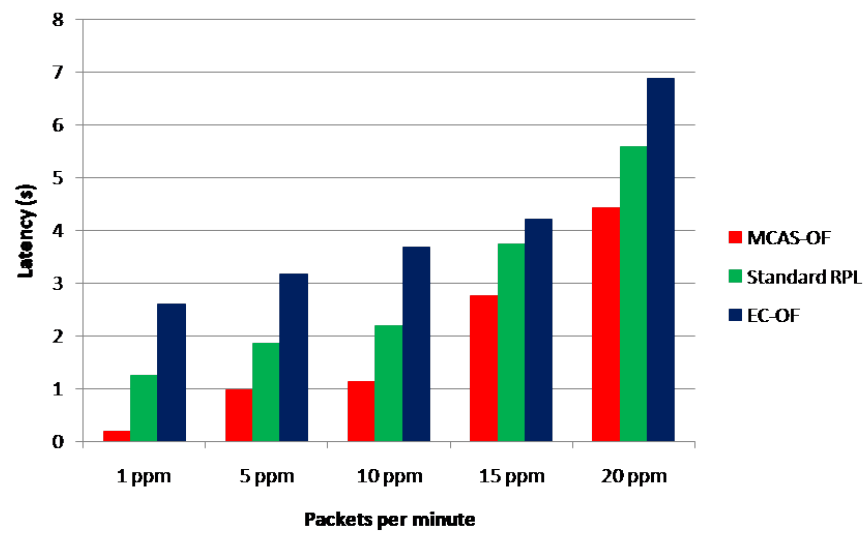

(a)

Indonesian J Elec Eng \& Comp Sci, Vol. 22, No. 1, April 2021 : 407 - 418 


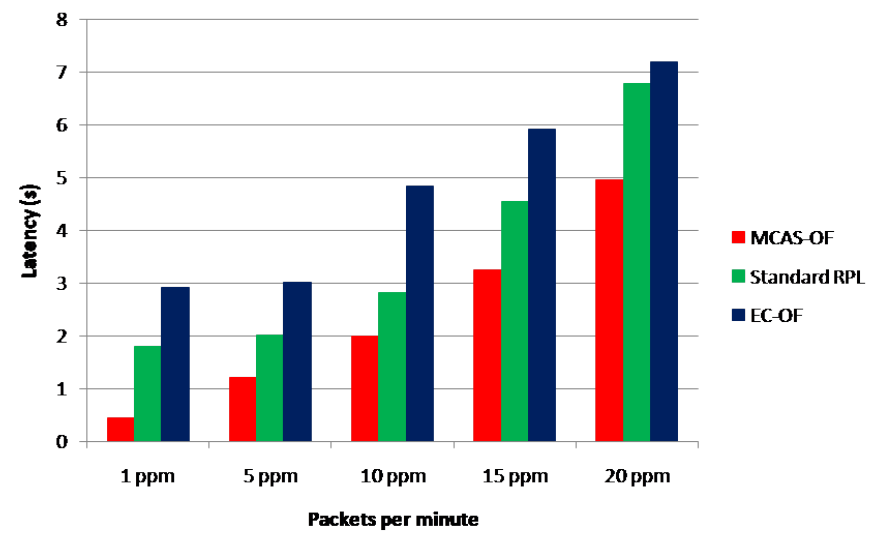

(b)

Figure 7. Latency vs number of packets per minute, (a) Density of 25 nodes, (b) Density of 50 nodes

\section{CONCLUSION}

Every IoT application has its requirements to be addressed through an appropriate protocols employment in different IoT layers. In our paper, we focused on the network layer, particularly the routing protocol RPL. There are many IoT applications in which the high packet transmissions needs to be reliable, nodes have to be power efficient while the network should be well balanced in terms of workload. Accordingly, we have proposed MCAS objective function, which addresses multi-constraints metrics, by taking into account radio signal strength indicator, hop count, energy consumption, and work-metric combined additively. The nodes process their ranks with the novel approach to select preferred parents. The network stability is also considered by an introduced adaptive threshold to limit the number of parents. Our evaluation results on IoT simulation environment COOJA confirmed that MCAS-OF outperforms Standart-RPL and EC-OF by improving considerably the PDR in high traffics for both densities with the rssi metric and workload balance between nodes, also it consumes less energy due to the hop count and energy metrics introduced during rank processing, achieves less latency thanks to congestion avoidance and minimize the standard deviation of node's power consumption. As part of future work, it is planned to test our modified RPL core in real skymote plateforms for a comparison achievement compared to simulations results and how networks with mobile nodes can affect our proposal.

\section{REFERENCES}

[1] I. Yaqoob, et al., "Internet of Things architecture: Recent advances, taxonomy, requirements, and open challenges," IEEE Wireless Communications, vol. 24, pp. 10-16, 2017.

[2] A. Ghasempour, "Internet of Things in Smart Grid: Architecture, Applications, Services, Key Technologies, and Challenges," Inventions journal, vol. 4, pp. 1-12, 2019.

[3] A. Musaddiq, et al., "A survey on resource management in IoT operating systems," IEEE Access, vol. 6, pp. 8459-8482, 2018.

[4] V. C. Gungor, et al., "Smart grid technologies: Communication technologies and standards," IEEE Transactions on Industrial Informatics, vol. 7, pp. 529-539, 2011.

[5] A. Ghasempour, "Advanced Metering Infrastructure in Smart Grid: Requirements, Challenges, Architectures, technologies, and Optimizations," Smart Grids: Emerging Technologies, Challenges and Future Directions,Ed. J. Lou, Nova Science Publishers, 2017.

[6] M. A. Naeem, et al., "A periodic caching strategy solution for the smart city in information-centric Internet of Things," Sustainability, vol. 10, pp. 2576, 2018.

[7] L. D. Xu, et al., "Internet of Things in industries: A survey," IEEE Transactions on Industrial Informatics, vol. 10, pp. 2233-2243, 2014.

[8] Y. A. Qadri, et al., "The Future of Healthcare Internet of Things: A Survey of Emerging Technologies" IEEE Communications Surveys and Tutorials, vol. 22, pp. 1121-1167, 2020. 
[9] R. Alexander, et al., "RPL: IPv6 Routing Protocol for Low-Power and Lossy Networks. Internet Engineering Task Force(IETF)" RFC 6550, 2012.

[10] A. E. Hassani, et al., "Assessment of a proactive routing protocol RPL in Ipv6 based wireless sensor networks" Third International Conference on Intelligent Computing in Data Sciences (ICDS), 2019.

[11] J. Granjal, et al., "Enabling network layer security on IPv6 wireless sensor networks" IEEE global telecommunications conference GLOBECOM, pp. 1-6, 2010.

[12] O. Gaddour, et al., "Simulation and performance evaluation of DAG construction with RPL" IEEE Third international conference on communications and networking, pp. 1-8, 2012.

[13] O. Gnawali, et al., "The Minimum Rank with Hysteresis Objective Function. Internet Engineering Task Force(IETF)" RFC 6719, 2012.

[14] P. Thubert, et al., "Objective Function Zero for the Routing Protocol for Low-Power and Lossy Networks (RPL). Internet Engineering Task Force(IETF)" RFC 6552, 2012.

[15] P. Sanmartin, et al., "Sigma routing metric for rpl protocol," Sensors, vol. 18, pp. 1277, 2018.

[16] O. Iova, et al., "Exploiting multiple parents in RPL to improve both the network lifetime and its stability," Proceeding of IEEE International Conference on Communications, pp. 610-616, 2015.

[17] P. O. Kamgueu, et al., "Energy-based routing metric for RPL," HAL archives, vol. 14, 2013.

[18] P. Gonizzi, et al., "Design and evaluation of a delay-efficient RPL routing metric," 9th International Wireless Communications and Mobile Computing Conference, 2013.

[19] K. S. Bhandari, et al., "A resource oriented route selection framework using contextual information based on fuzzy logic," Electronics, vol. 8, no. 9, pp. 1023, 2019.

[20] H. Lamaazi, et al., "OF-EC: A novel energy consumption aware objective function for RPL based on fuzzy logic," Journal of Network and Computer Applications, vol. 117, pp. 42-58, 2018.

[21] H. da S. Araújo, et al., "A proposal for IoT dynamic routes selection based on contextual information," Sensors, vol. 18, no. 2, pp. 1-16, 2018.

[22] S. Sankar, et al., "Fuzzy Logic Based Energy Aware Routing Protocol for Internet of Things," International Journal of Intelligent Systems and Applications, vol. 10, no. 10, pp. 11-19, 2018.

[23] Y. Chen, et al., "A scalable context-aware objective function(SCAOF) of routing protocol for agricultural low-power and lossy networks (RPAL)," Sensors, vol. 15, no. 8, pp. 19507-19540, 2015.

[24] A. E. Hassani, et al., "FTC-OF: Forwarding Traffic Consciousness Objective Function for RPL Routing Protocol," International Journal of Electrical and Electronic Engineering \& Telecommunications, 2020.

[25] A. E. Hassani, et al., "A New Objective Function Based on Additive Combination of Node and Link Metrics as a Mechanism Path Selection for RPL Protocol," International Journal of Communication Networks and Information Security, vol. 12, no. 1, pp. 63-68, 2020.

[26] J. Nassar, et al., "Multiple instances QoS routing in RPL: Application to smart grids," Sensors, vol. 18, pp. 1-16, 2018.

[27] S. N. Mishra, et al., "Eha-rpl: A composite routing technique in iot application networks," Advances in Intelligent Systems and Computing, vol. 1045, pp. 645-657, 2020.

[28] H. Al-Kashoash, et al., "Congestion-Aware Routing Protocol for 6LoWPANs," Congestion Control for 6LoWPAN Wireless Sensor Networks: Toward the Internet of Things, pp. 95-107, 2020.

[29] H. Lamaazi, et al., "A Comprehensive Survey on Enhancements and Limitations of the RPL protocol: A focus on the Objective Function," Ad Hoc Networks, vol. 96, 2019.

[30] A. Dunkels, et al., "Contiki - A lightweight and flexible operating system for tiny networked sensors," 29th annual IEEE international conference on local computer networks, pp. 455-462, 2004. 